\title{
Guest Editorial Special Issue on Wireless Sensor and Actuator Networks
}

W IRELESS Sensor Networks (WSNs), in their various shapes and forms, have greatly facilitated and enhanced the automated, remote, and intelligent monitoring of a large variety of physical systems. These networks consist of a large number of typically small devices, each incorporating sensing, processing, and wireless communications capabilities. Their use has penetrated a plethora of application domains from industrial and building automation, to environmental, wildlife, and health monitoring.

The control and systems community has played an important role in the maturing of WSNs, addressing issues related to their fundamental limits and designing strategies to optimize and control their operation so as to improve performance. Performance, for these systems, encompasses a variety of metrics that may vary with the application but in all cases includes the network's energy use which determines its usable lifetime. As WSN nodes are powered by small batteries, energy conservation has become a very important concern. Equally important, the existence of WSNs has provided a major application context to theoretical contributions of the control community including cooperative and distributed control, event-based monitoring, discrete-event systems, and consensus algorithms.

What is emerging as the next step in the WSN evolution is their use not only in monitoring, but also in controlling a physical system. To that end, some of the WSN nodes have to be augmented by integrating actuators. Actuators can be simple devices programmed to take immediate, one-shot, action in response to sensory input, or they can be more sophisticated entities (like robots) that interact with their environment in more complex ways. The resulting augmented version of WSNs is commonly referred to as Wireless Sensor and Actuator Networks (WSANs). WSANs are therefore heterogeneous networks that are comprised of networked sensor and actuator nodes communicating among each other via wireless links to perform distributed sensing and actuation tasks.

WSANs can be used to close loops over the network in a variety of applications, such as, environmental control, event detection and suppression, home automation, manufacturing, microclimate control, surveillance etc. The control systems community has recently made important contributions to understanding control over communication channels but this work has abstracted, for the most part, the communication medium. A new challenge is to consider a WSAN (with the emphasis on the N-the network) as the communications channel over which we seek to close control loops.

WSAN related research has attracted enough attention to spawn annual workshops and conferences, such as the Inter-

Digital Object Identifier 10.1109/TAC.2011.2164287 national Workshop on Wireless Sensor, Actuator and Robot Networks (WiSARN). Similar work has also found a good home at the IEEE Conference on Decision and Control which, over the last couple of years, hosted invited sessions on wireless sensor networks and distributed cooperative control and estimation. This issue appears to be the first special issue in a major journal to focus on these systems and so help illustrate the important contributions the control systems community is making in their evolution.

In response to the call for papers, we received 60 submissions from all over the world. After an initial screening, 9 submissions were declared "out of scope" (or with inappropriate technical content) and the remaining 51 were sent to reviewers. All manuscripts underwent a very rigorous peer review process. We finally selected 16 full papers and 7 technical notes for this special issue.

The full papers in this issue can be broadly organized into three main categories: (i) control with WSANs, (ii) control of WSANs, and (iii) WSAN node placement/deployment.

Papers in category (i) are concerned with performing certain tasks using a WSAN infrastructure/architecture. The paper by Notarstefano and Bullo and the one by Fischione consider optimization problems motivated or aided by a WSAN. The papers by You and Xie, Hendrickx et al. and Paschalidis and Li consider various issues related to distributed estimation/averaging and more general function computation over a WSAN. The paper by Pajic et al. considers the problem of stabilizing a plant over a rather general WSAN model.

Papers in category (ii) attack the problem of controlling WSANs to improve performance and efficiency from a variety of angles. The paper by Chen et al. presents a game-theoretic approach for channel allocation in WSANs. Alur et al. develop formal models for WSANs. The papers by Hariharan and Shroff and Nakayama et al. consider the problem of optimizing data gathering/aggregation by either devising optimal node coordination schemes (the former) or exploiting node mobility (the latter). The paper by Khouzani and Sarkar examines the vulnerability of a WSAN to specific types of attacks that lead to battery depletion.

Papers in category (iii) deal with network design/organization issues and, in particular, with WSAN node placement and deployment. The paper by Krause et al. considers node placement and scheduling from a combinatorial optimization perspective. Various forms of the optimal coverage problem are considered by Falcon et al. Razafindralambo and Simplot-Ryl, and Zhong and Cassandras. Finally, target tracking is considered in the paper by Zhang.

While their classification is not unique, the Technical notes in this issue fit in the three categories outlined above. Specifically, 
the papers by Mazo and Tabuada, D. Wu et al. and Mo et al. can be classified into category (i), the papers by $\mathrm{C}$. Wu et al. Kalathumani et al. and Caicedo and Zefran can be classified into category (ii), and, finally, the paper by Ghaffarkhah and Mostofi can be classified into category (iii).

It should be mentioned that a special issue simply provides a snapshot into the field taken at a particular point in time. Due to the standard page limitations of a Transactions volume, it can only include a relatively small number of papers. As a result, its coverage is by no means complete despite our best efforts.

Many individuals contributed to the success of this special issue. We take this opportunity to thank all the authors for their submissions. We are also indebted to a small army of referees who have put in the hard work and the long hours to review each paper in a timely and professional way. Editorial Assistant Elizabeth Kovács provided enormous and valuable assistance. Last, but not least, we are indebted to the past and current Editors-in-Chief, Christos Cassandras and Panos Antsaklis, respectively, for offering us this opportunity and for patiently waiting the completion of the special issue.
Jiming Chen

Zhejiang University

Hangzhou, China

Karl H. Johansson

Royal Institute of Technology

Stockholm, Sweden

Stephan Olariu

Old Dominion University

Norfolk, VA

Ioannis Ch. Paschalidis

Boston University

Boston, MA

Ivan Stojmenovic

University of Ottawa

Ottawa, ON Canada

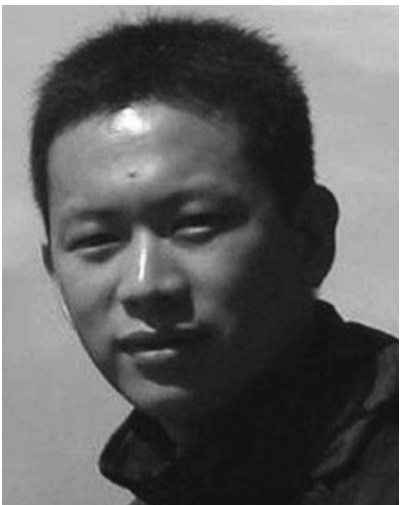

Jiming Chen (M'08-SM'11) received the B.Sc and Ph.D degrees in control science and engineering from Zhejiang University, Hangzhou, China, in 2000 and 2005, respectively.

He was a Visiting Researcher at INRIA in 2006, National University of Singapore, in 2007, University of Waterloo, from 2008 to 2010. Currently, he is a Full Professor with the Department of Control Science and Engineering, and the Coordinator of the Networked Sensing and Control Group, State Key Laboratory of Industrial Control Technology, Zhejiang University. He has published over 60 peer-reviewed papers. He currently serves as associate editor for several international Journals. He is the Guest Editor of , Computer Communication (Elsevier), Wireless Communication and Mobile Computer (Wiley) and the Journal of Network and Computer Applications (Elsevier). His research interests are estimation and control over sensor network, sensor and actuator network, target tracking in sensor networks, optimization in mobile sensor network.

Dr. Chen is a Guest Editor of the IEEE TRANSACTIONS ON AUTOMATIC CONTROL. He also serves as a co-Chair for Ad Hoc and Sensor Network Symposium, IEEE Globecom'11, General Symposia Co-Chair of ACM IWCMC'09 and ACM IWCMC'10, WiCON'10 MAC track co-Chair, IEEE MASS 2011 Publicity co-Chair, IEEE DCOSS' 11 Publicity co-Chair, IEEE ICDCS' 12 Publicity co-Chair and TPC member for IEEE ICDCS'10, IEEE MASS' 10 , IEEE SECON'11, IEEE INFOCOM'11, and IEEE INFOCOM'12.

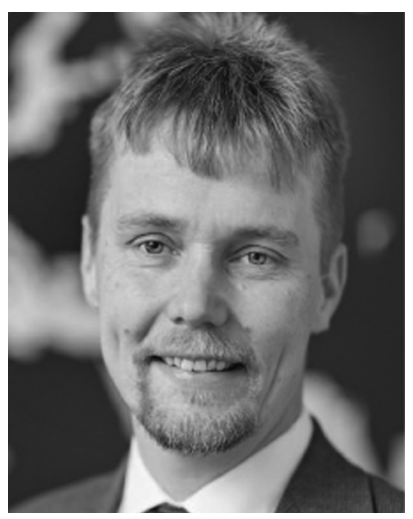

Karl H. Johansson (SM'08) received the M.Sc. and Ph.D. degrees in electrical engineering from Lund University, Lund, Sweden, in 1992 and 1997, respectively.

$\mathrm{He}$ is Director of the ACCESS Linnaeus Centre and Professor at the School of Electrical Engineering, Royal Institute of Technology, Sweden. He is a Wallenberg Scholar and has held a Senior Researcher Position with the Swedish Research Council. He has held visiting positions at UC Berkeley (1998-2000) and California Institute of Technology (2006-2007). He is the Chair of the IFAC Technical Committee on Networked Systems. He has served on the Executive Committees of several European research projects in the area of networked embedded systems. He is on the editorial board of IET Control Theory and Applications and the International Journal of Robust and Nonlinear Control. His research interests are in networked control systems, hybrid and embedded control, and control applications in automotive, automation and communication systems.

Dr. Johansson received an Individual Grant for the Advancement of Research Leaders from the Swedish Foundation for Strategic Research in 2005, the triennial Young Author Prize from IFAC in 1996, the Peccei Award from the International Institute of System Analysis, Austria, in 1993, and the Young Researcher Awards from Scania in 1996 and from Ericsson in 1998 and 1999. He was previously on the editorial board of the IEEE TRANSACTIONS on Automatic CONTROL and Automatica. 


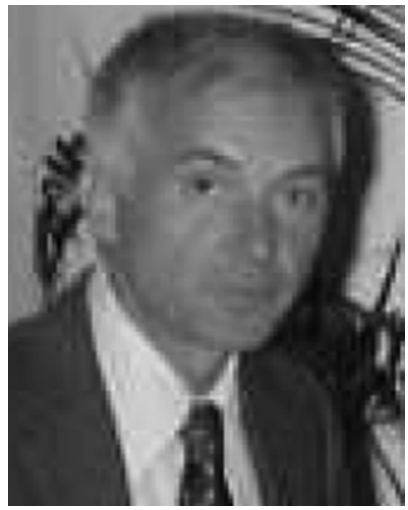

Stephan Olariu has held many different roles and responsibilities as a member of numerous organizations and teams. Much of his experience has been with the design and implementation of robust protocols for wireless networks and in particular sensor networks and their applications. He is a world-renowned technologist in the areas of wireless networks, mobile multimedia systems, parallel and distributed systems, parallel and distributed architectures and networks. He was invited and visited more than 120 universities and research institutes around the world lecturing on topics ranging from wireless networks and mobile computing, to biology-inspired algorithms and applications, to telemedicine, to wireless location systems, and security. He is the Director of the Sensor Networks Research Group, Old Dominion University. He has coauthored/edited four books including Solutions to Parallel and Distributed Computing Problems: Lessons from Biological Sciences (Wiley, 2001), Numerical Simulations and Case Studies (Wiley , 2005), and the Handbook of Bio-inspired Algorithms and Applications. He has published over 200 articles in archival journals and over 200 papers in conference proceedings. He is an Associate Editor of Networks and serves on the editorial board of the Journal of Parallel and Distributed Computing, Ad Hoc and Sensor Networks, and the International Journal of Parallel, Emergent and Distributed Systems. His research interests are in the area of complex systems enabled by large-scale deployments of sensors and more specifically in securing systems of systems.

Dr. Olariu is an Associate Editor of the IEEE TRANSACTIONS ON PARALLEL AND DisTRIBUTED SYSTEMS.

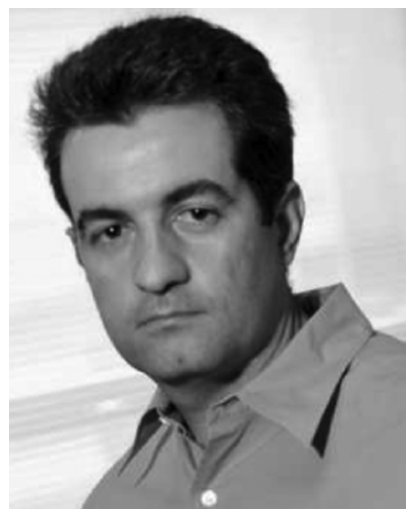

Ioannis Ch. Paschalidis (SM'06) received the M.S. and Ph.D. degrees in electrical engineering and computer science from the Massachusetts Institute of Technology (MIT), Cambridge, in 1993 and 1996, respectively.

He is a Professor in the College of Engineering, Boston University, Boston, MA, with a joint appointment in the Department of Electrical and Computer Engineering and the Division of Systems Engineering. He is a Co-Director of the Center for Information and Systems Engineering (CISE), Boston University. He is also affiliated with the BioMolecular Engineering Research Center (BMERC) and serves as the Academic Director of the Sensor Network Consortium (SNC) (an industry consortium he spearheaded). He is an Associate Editor of the SIAM Journal on Control and Optimization and the ACM Transactions on Sensor Networks and was an Associate Editor of Operations Research Letters. His research interests are fields systems and control, networking, applied probability, optimization, operations research, computational biology, and bioinformatics. His recent work has found applications in communication and sensor networks, protein docking, logistics, cyber-security, robotics, the smart-grid, and finance.

Dr. Paschalidis received a CAREER Award (2000) from the National Science Foundation and the Second Prize in the 1997 George E. Nicholson Paper Competition by INFORMS, and was an Invited Participant at the 2002 Frontiers of Engineering Symposium. He was an Associate Editor of the IEEE TRANSACTIONS ON AUTOMATIC CONTROL.

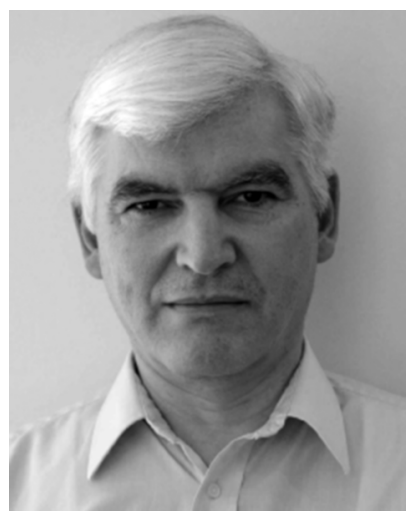

Ivan Stojmenovic (F'08) received the Ph.D. degree in mathematics.

He held regular and visiting positions in Serbia, Japan, U.S., Canada, France, Mexico, Spain, U.K. (as Chair in Applied Computing at the University of Birmingham), Hong Kong, Brazil, Taiwan, and China. He is a Full Professor at the University of Ottawa, Ottawa, ON, Canada and an Adjunct Professor at the University of Novi Sad, Novi Sad, Serbia. He published over 250 different papers, and edited seven books on wireless, Ad Hoc, sensor and actuator networks and applied algorithms with Wiley. He is editor of over one dozen journals, and Founder and Editor-in-Chief of three journals (MVLSC, IJPEDS, and AHSWN). He is one of about 300 computer science researchers (with h-index at least 40) and has more than 10000 citations.

Dr. Stojmenovic received three Best Paper Awards and the Fast Breaking Paper for October 2003, by Thomson ISI ESI, the Royal Society Research Merit Award, UK. , the IEEE CS Distinguished Visitor, 2010-2012, and the Excellence in Research Award of the University of Ottawa in 2009. He is Editor-in-Chief of the IEEE TRANSACTIONS ON PARALLEL AND DISTRIBUTED SYSTEMS (from January 2010). He chaired and/or organized more than 60 workshops and conferences, and served in more than 200 program committees. He was program co-chair at IEEE PIMRC'08, IEEE AINA'07, IEEE MASS'04\&'07, EUC'05\&'08-'10, AdHocNow'08, IFIP WSAN'08, WONS'05, MSN'05\&'06, ISPA'05\&'07, founded the workshop series at IEEE MASS, ICDCS, DCOSS, WoWMoM, ACM Mobihoc, IEEE/ACM CPSCom, FCST, MSN, and is/was Workshop Chair at IEEE INFOCOM'11, IEEE MASS'09, ACM Mobihoc'07 and '08. 